\title{
Complexity Analysis of EEG Signals
}

\author{
Yue-Der Lin, Shing-Ming Sung *, Fok-Ching Chong, Te-Son Kuo and Chi-Hung Liu \\ Department of Electrical Engineering, National Taiwan University \\ * Department of Neurology, Taipei Municipal Jen-Ai Hospital
}

\section{ABSTRACT :}

Electroencephalogram (EEG) is known to be a complex time series signal and also is important in clinical neurophysiology. Here the technique of easily calculable measure of complexity $C$ was applied to the analysis of EEG signals. Sixteen-channel EEG signals of 20 normal and 20 apoplectic subjects between 55 and 78 years of age were measured monopolarly according to the international tentwenty system with the ipsilateral ear (Al or A2) as the reference. The results show that the $C$ values for the normal population are significantly higher than those of the apoplectic population at 0.01 confidence level for all channels and we propose that complexity $\mathrm{C}$ could help determine if EEG is normal or abnormal.

Keywords: Electroencephalogram(EEG), Complexity,International ten-twenty system

\section{INTRODUCTION :}

The calculation of complexity is one of the quantity measure for a chaotic time series. For a nonlinear dynamical system, a symbolic sequence may be obtained which represents its dynamic behavior. Roughly speaking, the complexity of this symbolic sequence is a measure on the extent to which the given sequence resembles a random one. Kolmogorov proposed that the complexity of a given string composed of zeros and ones is given by the number of bits of the shortest computer program which can generate this string[1]. In this study, the algorithm for complexity $C$ which has been introduced by Kaspar and Schuster is adopted for the reasons of being easily implemented and high speed. Kaspar and Schuster have also shown that, for a time series, complexity $C$ is a finer measure for order than the Lyapunov exponents[2]. Our main intention is to investigate whether tne measure of complexity $C$ is suitable for $E E G$ analysis in clinical application or not.

\section{METHODS AND MATERIALS :}

Twenty healthy subjects and twenty apoplectic subjects (all were confirmed by two or three experienced clinical doctors) took part in this study (Table I). The age ranges of the subjects are chosen to be as close as possibie. All EEGs were recorded in a quiet, dimly lit room where the subjects being wakeful were seated comfortablely with their eyes closed. $\mathrm{Ag} / \mathrm{AgCl}$ cup electrodes were fixed to the scalp at points Fp1 , F3, C3, P3, O1, Fp2, F4, C4, P4, O2, F7, T3, T5, F8, T4 and $T 6$ of the international ten-twenty system [3] with the ipsilateral ear electrode ( $\mathrm{A} 1$ or $\mathrm{A} 2$ ) as the reference. The 16-channel EEGs were recorded by an electroencephalograph (Nihon Kohden ZE-431 A, $35 \mathrm{~Hz}$ low-pass filter, $48 \mathrm{~dB} /$ octave) and the analog signals were digitized by an A/D card (Data Translation, Data Acquisition Board DT-2801, 12-bit resolution) at a sampling rate of $128 \mathrm{~Hz}$ to satisfy the requirement of sampling theorem. One segment of artefact-free EEG of 15.6 seconds long (at least) for each subject was selected to the computation of complexity. The algorithm of complexity we used is that introduced by Kaspar and Schuster[2] and the flowchart is shown in Fig. 1, which has been modified from the original one.

\section{RESULT :}

The statistical data of the normal and apoplectic group are shown in Table II. By statistical analysis, the null hypothesis of equal variances of $\mathrm{C}$ for normal and apoplectic population is accepted ( $p>0.01$ for each channel, two-sided $f$-test), and the average $C$ values are higher in healthy population than in apoplectic population ( $p<0.01$ for each channel, one-sided t-test)

\section{DISCUSSION :}

The benefits of measuring $C$ are the higher speed and easy implementation. For the EEG data of one subject, the 16-channel results could be derived in several seconds using a 486PC DX-66 machine. The running time and the storage space in calculating $C$ are proportional to $n$, where $n$ is the sequence length. Besides, there are no parameters needed to be determined.

A simpler approach to analyze EEG is presented in this study, and the measure of 
complexity $C$ seems suitable for EEG analysis in clinical application as there exists significant difference of $\mathrm{C}$ between normal and apoplectic group. Besides, from the result of Table II, the variances in apoplectic group are larger than those in normal group. This is because the foci of the apoplectic subjects in this study are not identical, thus makes the larger variance in apoplectic group

In addition to providing another useful feature for EEG diagnosis, the possible applications of measuring $\mathrm{C}$ may include the research on the change of EEG in different mental tasks, the monitoring of sleep stages or anesthesia levels, and information processing of the brain.

\section{REFERENCE}

[1] A.V.Kolmogorov, "Three approaches to the quantitative definition of information", Probl Inform. Transmission, 1965, vol.1, pp.1-7.

[2] F.Kaspar and H.G.Schuster, "Easily calculable measure for the complexity of spatiotemporal patterns", Phys. Rev. A, 1987, vol.36, pp.842-845.

[3] H.H.Jaspar, "The ten-twenty electrode system of the International Federation", Electroenceph. clin. Neurophysiol., 1958, vol.10, pp. 371-375.

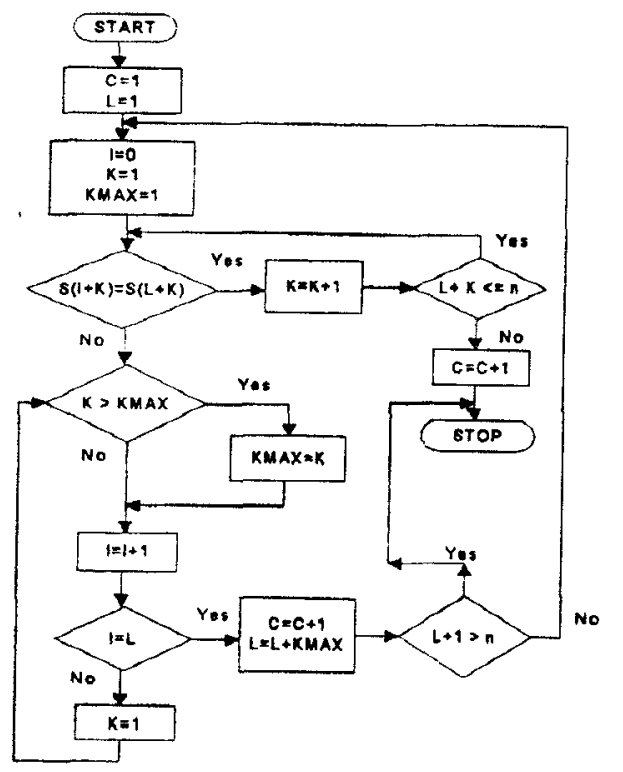

Fig. 1 The flowchart of calculating complexity C.
Table I About the subject number and subject age in this study.

\begin{tabular}{|c|c|c|c|c|}
\hline \multirow{2}{*}{ Group } & \multirow{2}{*}{ Number of Subject } & \multicolumn{3}{|c|}{ Age (Year) } \\
\hline & & Mean & S.D. & Range \\
\hline NM & 6 & 68.83 & 5.78 & $59-75$ \\
\hline NE & 14 & 67.57 & 7.37 & $55-78$ \\
\hline $\mathrm{AM}$ & 10 & 66.90 & 6.23 & $57-76$ \\
\hline $\mathrm{AF}$ & 10 & 68.30 & 6.09 & $60-78$ \\
\hline $\mathrm{NM}+\mathrm{NF}$ & 20 & 67.35 & 6.78 & $55-78$ \\
\hline$A M+A F$ & 20 & 67.60 & 6.04 & $57-78$ \\
\hline
\end{tabular}

Legend :

NM : Normal Male

NF : Normal Female

AM : Apoplectic Male

AF : Apoplectic Female

Table II The statistical values of $C$ at 16 scalp positions for normal and apoplectic group.

\begin{tabular}{ccc}
\hline Positions & Normal $(\mathrm{n}=20)$ & Apoplectic $(\mathrm{n}=20)$ \\
\hline Fp1 & $0.55 \pm 0.11$ & $0.37 \pm 0.11$ \\
F3 & $0.69 \pm 0.05$ & $0.50 \pm 0.10$ \\
C3 & $0.69 \pm 0.04$ & $0.50 \pm 0.13$ \\
P3 & $0.68 \pm 0.04$ & $0.51 \pm 0.10$ \\
O1 & $0.65 \pm 0.04$ & $0.48 \pm 0.13$ \\
Fp2 & $0.56 \pm 0.11$ & $0.35 \pm 0.12$ \\
F4 & $0.66 \pm 0.04$ & $0.50 \pm 0.10$ \\
C4 & $0.69 \pm 0.05$ & $0.52 \pm 0.08$ \\
P4 & $0.66 \pm 0.04$ & $0.51 \pm 0.08$ \\
O2 & $0.64 \pm 0.04$ & $0.47 \pm 0.11$ \\
F7 & $0.67 \pm 0.07$ & $0.47 \pm 0.13$ \\
T3 & $0.74 \pm 0.06$ & $0.50 \pm 0.13$ \\
T5 & $0.67 \pm 0.06$ & $0.49 \pm 0.11$ \\
F8 & $0.65 \pm 0.07$ & $0.46 \pm 0.09$ \\
T4 & $0.74 \pm 0.08$ & $0.48 \pm 0.12$ \\
T6 & $0.66 \pm 0.07$ & $0.50 \pm 0.11$ \\
\hline
\end{tabular}

